Chapter 9

\title{
Generalized Path Integral Technique: Nanoparticles Incident on a Slit Grating, Matter Wave Interference
}

\author{
Valeriy I. Sbitnev \\ Additional information is available at the end of the chapter \\ http://dx.doi.org/10.5772/53471
}

\section{Introduction}

One of the crises of contemporary mathematics belongs in part to the subject of the infinite and infinitesimals [1]. It originates from the barest necessity to develop a rigorous language for description of observable physical phenomena. It was a time when foundations of integral and differential calculi were developed. A theoretical foundation for facilitation of understanding of classical mechanics is provided by the concepts of absolute time and space originally formulated by Sir Isaac Newton [2]. Space is distinct from body. And time passes uniformly without regard to whether anything happens in the world. For this reason Newton spoke of absolute space and absolute time as of a "container" for all possible objects and events. The space-time container is absolutely empty until prescribed metric and a reference frame are introduced. Infinitesimals are main tools of differential calculus [3, 4] within chosen reference frames.

Infinitesimal increment being a cornerstone of theoretical physics has one receptee default belief, that increment $\delta V$ tending to zero contains a lot of events to be under consideration. Probability of detection of a particle within this infinitesimal volume $\rho(\vec{r}) \delta V$ is adopted as a smooth differentiable function with respect to its argument. From experience we know that for reproducing the probability one needs to accumulate enormous amount of events occurring within this volume. On the other hand we know, that as $\delta V$ tends to zero we lose information about amount of the events. What is more, the information becomes uncertain. It means the infinitesimal increment being applied in physics faces with a conflict of depth of understanding physical processes on such minuscule scales. This trouble is avoided in quantum mechanics by proclamation that infinitesimal increments are operators, whereas observables are averaged on an ensemble. 
In light of classical views Newton maintained the theory that light was made up of tiny particles, corpuscles. They spread through space in accordance with law of classical mechanics. Christian Huygens (a contemporary of Newton), believed that light was made up of waves vibrating up and down perpendicularly to direction of the light propagation. It comes into contradiction with Newtonian idea about corpuscular nature of light. Huygens was a proclaimer of wave mechanics as opposite to classical mechanics [5].

We abstain from allusion to physical vacuum but expand Huygensian idea to its logical completion. Let us imagine that all Newtonian absolute space is not empty but is populated everywhere densely by Huygensian vibrators. The vibrators are silent at absence of wave propagating through. But as soon as a wave front reaches some surface all vibrators on this surface begin to radiate at a frequency resonant with that of incident wave. From here it follows, that in each point of the space there are vibrators with different frequencies ranging from zero frequency up to infinite. All are silent in absence of an external wave perturbance. Thus, the infinitesimal volume $\delta V$ is populated by infinite amount of the vibrators with frequencies ranging from zero to infinity. They are virtual vibrators facilitating propagation of waves through space.

Let us return to our days. One believes that besides matter and physical fields there is nothing more in the universe. Elementary particles are only a building material of "eternal and indestructible" substance of the cosmos. However we should avow that all observed matter and physical fields, are not the basis of the world, but they are only a small part of the total quantum reality. Physical vacuum in this picture constitutes a basic part of this reality. In particular, modern conception of the physical vacuum covers Huygens's idea perfectly. All space is fully populated by virtual particle-antiparticle pairs situated on a ground level. Such a particle-antiparticle pair has zero mass, zero charge, and zero magnetic moment. Famous Dirac' sea (Dirac postulated that the entire universe is entirely filled by particles with negative energy) is boundless space of electron-positron pairs populated everywhere densely - each quantum state possessing a positive energy is accompanied by a corresponding state with negative energy. Electron has positive mass and positron has the same mass but negative; electron has negative charge and positron has the same positive charge; when electron and positron dance in pair theirs magnetic moments have opposite orientations, so magnetic moment of the pair is zero.

Let electron and positron of a virtual pair rotate about mass center of this pair. Rotation of the pairs happens on a Bohr orbit. Energy level of the first Bohr orbit, for example, $m v^{2} / 2$, is about $14 \mathrm{eV}$. Here $m$ is electron mass and $v$ is its velocity (on the first Bohr orbit the velocity is about $2.18810^{6} \mathrm{~m} / \mathrm{s}$ ). Energy of the pair remains zero since positron has the same energy but with negative sign. Quantum fluctuations around this zero energy are as zero oscillations of electromagnetic field. Observe that, energy releasing of electron and positron from vacuum occurs at $m c^{2}=1.022 \mathrm{MeV}$. So we see that there are about $7.510^{4}$ Bohr orbits lying below this energy. That is, there is a vast scope for occupation of different Bohr orbits by the virtual electron-positron pairs.

A short outline given above is a basis for understanding of interference effects to be described below. 
Ones suppose that random fluctuations of electron-positron pairs take place always. What is more, these fluctuations are induced by other pairs and by particles traveling through this random conglomerate. Edward Nelson has described mathematical models [6, 7] representing the above random fluctuations as Brownian motions of particles that are subjected by random impacts from particles populating aether (Nelson's title of a lower environment). The model is viewed as a Markov process

$$
d x(t)=b(x(t), t) d t+d w(t)
$$

loaded by a Wiener term $w$ with diffusion coefficient equal to $\hbar / 2 m$, where $m$ is a mass of the particle and $\hbar$ is the reduced Planck constant. In this perspective Nelson has considered two Markov processes complementary to each other. One is described by forward-difference operator in the time, here $b(x(t), t)$ is a velocity calculated forward. And other equation is described by backward-difference operator in the time with the velocity $b^{+}(x(t), t)$ calculated backward. In general $b(x(t), t) \neq b^{\dagger}(x(t), t)[6,7]$. The two complementary processes, by means of transition to two new variables, real and imaginary, finally lead to emergence of the Schrödinger equation.

Nelson' vision that aether fluctuations look as Brownian movements of subparticles with $\hbar / 2 m$ being the diffusion coefficient of the movements, correlates with Feynman's ideas about quantum fluctuations of virtual particles in vacuum [8]. The Feynman path integral is akin to the Einstein-Smoluchowski integral equation [9]. The latter computes transition probability density. We shall deal with the modified Feynman path integral loaded by a temperature multiplied by the Boltzmann constant. At that, probability amplitude stays as a fundamental mathematical object at all stages of computations. As an example we shall consider emergence of interference patterns at scattering heavy particles on gratings. The particles are heavy in the sense that they adjoin to both realms, quantum and classical. They are nanoparticles. Such nanoparticles have masses about $100 \mathrm{amu}$ and more, as, for example, fullerene molecules [10] shown in Fig. 1. It is remarkable that there are many experiments with such molecules showing interference patterns in the near field [11-18]. On the other hand these molecules are so large, that they behave themselves as classical particles at ordinary conditions.

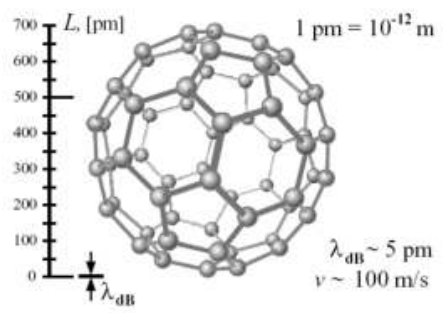

Figure 1. The fullerene molecule $C_{60}$ consists of 60 carbon atoms. Its radius is about $700 \mathrm{pm}$. De Broglie wavelength of the molecule, $\lambda_{\mathrm{dB}}$, is about $5 \mathrm{pm}$ at a flight velocity $v=100 \mathrm{~m} / \mathrm{s}$ [18]. The molecules are prepared in a thermal emission gun which has temperature about $1000 \mathrm{~K}$. It means that carbon atoms accomplish thermal fluctuations. 
The article consists of five sections. Sec. 2 introduces a general conception of the path integral that describes transitions along paths both of classical and quantum particles. Here we fulfill expansions in the Taylor series of terms presented in the path integral. Depending on type of presented parameters we disclose either the Schrödinger equation or diffusion-drift equation containing extra term, osmotic diffusion. In the end of the section we compute passing nanoparticles through $N$-slit grating. Sec. 3 deals with interference patterns from the $\mathrm{N}$-slit grating. Specifically, we study blurring of the Talbot carpet (an interference pattern emergent under special conditions imposed on the grating $[19,20])$ arising under decoherence of incident on the grating nanoparticles. In Sec. 4 we find equations for computing the Bohmian trajectories. Also we compute variance of momenta along the trajectories. These computations lead to emergence of the uncertainty conditions. In concluding Sec. 5 we summarize results. For confirmation of existence of the Bohmian trajectories here we mention interference experiments with single silicon oil droplet [21].

\section{Generalized path integral}

Let many classical particles occupy a volume $V$ and they move with different velocities in different directions. Let us imagine that there is a predominant orientation along which ensemble of the particles drifts. As a rule, one chooses a small volume $\delta V$ in order to evaluate such a drift, Fig. 2. Learning of statistical mechanics begins with assumption that the volume should contain many particles.

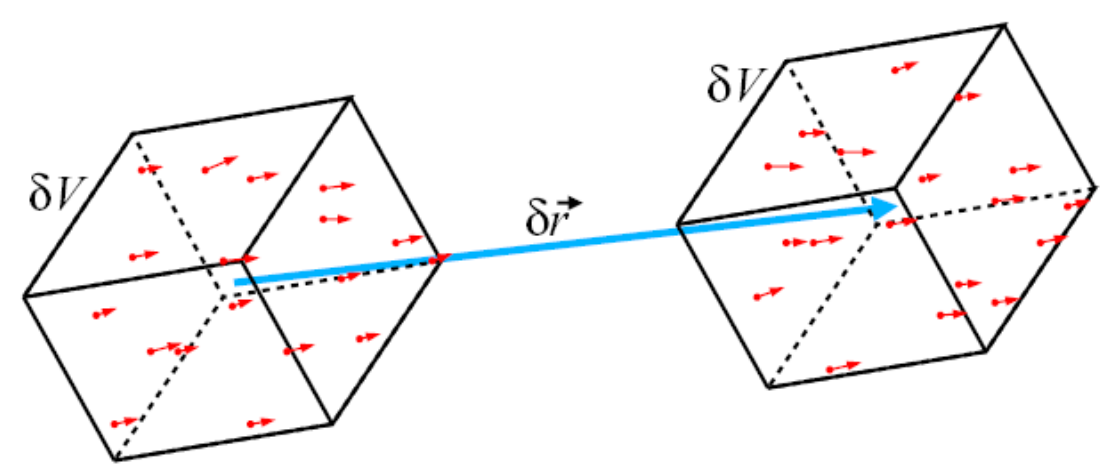

Figure 2. Infinitesimal volume $\delta V$ contains many particles moving with different velocities having predominant orientation along blue arrow. The infinitesimal volume $\delta V$, as a mental image, is shifted along the same orientation.

The problem is to find transition probabilities that describe transition of the particle ensemble from one statistical state to another. These transient probabilities can be found through solution of the integral Einstein-Smoluchowski equation [9]. This equation in mathematical physics is known as Chapman-Kolmogorov [22-24] equation.. This equation looks as follows 


$$
p\left(\vec{r}_{2}, \vec{r}_{1} ; t+\delta \tau\right)=\int_{R} p\left(\vec{r}_{2}, \vec{r} ; t+\delta \tau, t\right) p\left(\vec{r}, \vec{r}_{1} ; t\right) d V
$$

This equation describes a Markovian process without memory. That is, only previous state bears information for the next state. Integration here is fulfilled over all a working scene R, encompassing finite space volume. Infinitesimal volume $\delta V$ in the integral tends to zero. It should be noted, however, that this volume should contain as many particles as possible for getting a satisfactory statistical pattern. One can see that this claim enters in conflict with the assumption $\delta V \rightarrow 0$.

Next we shall slightly modify approach to this problem. Essential difference from the classical probability theory is that instead of the probabilities we shall deal with probability amplitudes. The transition amplitudes can contain also imaginary terms. They bear information about phase shifts accumulated along paths. In that way, a transition from an initial state $\vec{q}_{0}$ to a final state $\vec{q}_{1}$ through all intermediate positions $\vec{q}_{x}$ given on a manifold $\mathrm{R}^{3}$ (see Fig. 2) is represented by the following path integral

$$
\psi\left(\vec{q}_{1}, \vec{q}_{0} ; t+\delta t\right)=\int_{R} K\left(\vec{q}_{1}, \vec{q}_{x} ; t+\delta t, t\right) \psi\left(\vec{q}_{x}, \vec{q}_{0} ; t\right) D^{3} q_{x} .
$$

Here function $\psi(\ldots)$ is a probability amplitude. Probability density $p(\ldots)$, in turn, is represented by square of modulo of the probability amplitude, namely, $p(\ldots)=|\psi(\ldots)|^{2}$. Integral kernel $K\left(\vec{q}_{1}, \vec{q}_{x} ; t+\delta t, t\right)$ represents the transition amplitude from an intermediate state $\vec{q}_{x}$ to a final state $\vec{q}_{1}$. It is called propagator $[8,25,26]$. We suppose that the propagator has the following standard form

$$
K\left(\vec{q}_{1}, \vec{q}_{x} ; t+\delta t, t\right)=\frac{1}{A} \exp \left\{-\frac{L\left(\vec{q}_{x}, \dot{\vec{q}}_{x}\right) \delta t}{\Gamma}\right\},
$$

where denominator $\Gamma$ under exponent is a complex-valued quantity, i.e., $\Gamma=\beta+\mathbf{i} \hbar$. The both parameters, $\beta$ and the reduced Planck constant $\hbar$, have dimensionality of energy multiplied by time. From here it follows that $\beta=2 k_{\mathrm{B}} T \delta t$. Here $k_{\mathrm{B}}$ is Boltzmann constant and $T$ is temperature. So, we can write

$$
\Gamma=2 k_{\mathrm{B}} T \delta t+\mathbf{i} \hbar
$$

Factor 2 at the first term is conditioned by the fact that the kernel $K$ relates to transitions of the probability amplitude $\psi$, not the probability $p$. Observe that a fullerene molecular beam 
for interference experiment prepared in a Knudsen cell at T=1070 K [27] spreads further in a vacuum. That is, fullerene molecules keep this temperature. From here it follows, that thermal fluctuations of carbon atoms from equilibrium occur at that temperature as the fullerene molecules propagate further within the vacuum chamber. One can see that at $T=1000 \mathrm{~K}$ the term $2 k_{\mathrm{B}} T \delta t$ may be about $\hbar$ if $\delta t$ is about $10^{-14} \mathrm{~s}$.

Next let us imagine that particles pass through a path length one by one. That is, they do not collide with each other along the path length. The particles are complex objects, however. They are nanoparticles. Fullerene molecule, for example, contains 60 carbon atoms, Fig. 1. Conditionally we can think that the atoms are connected with each other by springs simulating elastic vibrations. In this view the Lagrangian $L\left(\vec{q}_{x}, \vec{q}_{x}\right)$ can be written in the following form

$$
L\left(\vec{q}_{x}, \dot{\vec{q}}_{x}\right)=\sum_{k=1}^{N}\left[\frac{m}{2} \frac{\left.\left(\vec{q}_{1, k}-\vec{q}_{x, k}\right)\right)^{2}}{\delta t^{2}}-U\left(\vec{q}_{x, k}\right)\right] .
$$

Here $N$ is amount of atoms, constituent complex molecule, and $m$ is mass of a single atom. By supposing that there are no quantum permutations between atoms we may expand the Lagrangian further

$$
\begin{aligned}
L\left(\vec{q}_{x}, \dot{\vec{q}}_{x}\right) & =\frac{m}{2} \sum_{k=1}^{N} \frac{\left(\vec{q}_{1, k}-\vec{q}_{1}+\vec{q}_{1}-\vec{q}_{x, k}+\vec{q}_{x}-\vec{q}_{x}\right)^{2}}{\delta t^{2}}-\sum_{k=1}^{N} U\left(\vec{q}_{x, k}\right) \\
& =\frac{m_{N}}{2} \frac{\left(\vec{q}_{1}-\vec{q}_{x}\right)^{2}}{\delta t^{2}}-m_{N} \frac{\left(\vec{q}_{1}-\vec{q}_{x}\right)}{\delta t} \frac{1}{N} \sum_{k=1}^{N} \frac{\left(\vec{\delta}_{1, k}-\vec{\delta}_{x, k}\right)}{\delta t}+\frac{m}{2} \sum_{k=1}^{N} \frac{\left(\vec{\delta}_{1, k}-\vec{\delta}_{x, k}\right)^{2}}{\delta t^{2}}-\sum_{k=1}^{N} U\left(\vec{q}_{x, k}\right) .
\end{aligned}
$$

Here we admit that $\vec{q}_{x}$ and $\vec{q}_{1}$ are coordinates of center of mass of that complex molecule in intermediate and final positions. And $m_{N}=N m$ is a mass of the molecule referred to its center of mass. Small deviations $\vec{\delta}_{1, k}=\left(\vec{q}_{1}-\vec{q}_{1, k}\right)$ and $\vec{\delta}_{x, k}=\left(\vec{q}_{x}-\vec{q}_{x, k}\right)$ are due to oscillations of the $k$ th atoms with respect to the center of mass.

Let us consider the second row in Eq. (7). First of all we note that the term $\left(\vec{q}_{1}-\vec{q}_{x}\right) / \delta t$ represents a velocity of movement of the center of mass. In such a case the first term is a kinetic energy of the center of mass. The second term represents product of the center mass velocity on an averaged velocity of partial oscillations of atoms constituting this molecule. The averaged velocity we believe vanishes because of conservation of total momentum. The third term represents a thermal kinetic energy of partial oscillating atoms constituting this molecular object. This energy is small enough. But it is sufficient to exhibit itself in the Casimir effect. The last term is a total potential energy $U\left(\vec{q}_{x}\right)$ in the point $\vec{q}_{x}$. 
The path integral (3) contains functions depending only on coordinates $\vec{q}_{0}, \vec{q}_{1}, \vec{q}_{x}$ relating to positions of the center of mass. Whereas the Lagrangian (6) gives description for behavior of each atom constituent the complex molecule. Here we shall suppose that oscillations of all atoms are noncoherent. And consequently they do not give contribution to interference effect on output. We believe that these oscillations provide a thermal noise. And next we shall replace this oscillating background by a corresponding thermal term. For this reason, we believe that along with the reduced Planck constant $\hbar$ the parameter $2 k_{\mathrm{B}} T \delta t$ in Eq. (5) can be different from zero as well.

\subsection{Expansion of the path integral}

The next step is to expand terms, ingoing into the integral (3), into Taylor series. The wave function written on the left is expanded up to the first term

$$
\psi\left(\vec{q}_{1}, \vec{q}_{0} ; t+\delta t\right) \approx \psi\left(\vec{q}_{1}, \vec{q}_{0} ; t\right)+\frac{\partial \psi}{\partial t} \delta t .
$$

As for the terms under the integral, here we preliminarily make some transformations. We define a small increment

$$
\vec{\xi}=\vec{q}_{1}-\vec{q}_{x} \Rightarrow D^{3} q_{x}=-D^{3} \xi
$$

The Lagrangian (7) is rewritten, in such a case, in the following view

$$
L\left(\vec{q}_{x}, \dot{\vec{q}}_{x}\right)=\frac{m_{N}}{2} \frac{\xi^{2}}{\delta t^{2}}-m_{N} \frac{\xi}{\delta t} \frac{1}{N} \sum_{k=1}^{N} \frac{\left(\vec{\delta}_{1, k}-\vec{\delta}_{x, k}\right)}{\delta t}+\frac{m}{2} \sum_{k=1}^{N} \frac{\left(\vec{\delta}_{1, k}-\vec{\delta}_{x, k}\right)^{2}}{\delta t^{2}}-U\left(\vec{q}_{x}\right)
$$

Here $U\left(\vec{q}_{x}\right)$ is sum of all potentials $U\left(\vec{q}_{x, k}\right)$ given in the center of mass. Further we shall deal with the path integral (3) where the kernel $K$ contains the Lagrangian given from Eq. (10). The under integral function $\psi\left(\vec{q}_{x}, \vec{q}_{0} ; t\right)=\psi\left(\vec{q}_{1}-\vec{\xi}, \vec{q}_{0} ; t\right)$ is subjected to expansion into the Taylor series up to the second terms of the expansion

$$
\psi\left(\vec{q}_{1}-\vec{\xi}, \vec{q}_{0} ; t\right) \approx \psi\left(\vec{q}_{1}, \vec{q}_{0} ; t\right)-(\nabla \psi, \vec{\xi})+\nabla^{2} \psi \cdot \xi^{2} / 2
$$

The potential energy $U\left(\vec{q}_{x}\right)=U\left(\vec{q}_{1}-\vec{\xi}\right)$ is subjected to expansion into the Taylor series by the small parameter $\vec{\xi}$ also. Here we restrict themselves by the first two terms of the expansion, $U\left(\vec{q}_{1}\right)-\left(\nabla U\left(\vec{q}_{1}\right), \vec{\xi}\right)$.

Taking into account the expressions (8)-(11) and substituting theirs into Eq. (3) we get 


$$
\begin{aligned}
\psi\left(\vec{q}_{1}, \vec{q}_{0} ; t\right)+\frac{\partial \psi}{\partial t} \delta t & =-\frac{1}{A} \int_{R^{3}} \exp \left\{-\frac{1}{\Gamma}(\frac{m_{N}}{2} \frac{\xi^{2}}{\delta t}-\underbrace{\xi \underbrace{N}_{N} \sum_{k=1}^{N} \frac{\left(\vec{\delta}_{1, k}-\vec{\delta}_{x, k}\right)}{\delta t}}_{(a)}+\underbrace{\left[\frac{m}{2} \sum_{k=1}^{N} \frac{\left(\vec{\delta}_{1, k}-\vec{\delta}_{x, k}\right)^{2}}{\delta t^{2}}\right]}_{(b)} \delta t\right. \\
& \left.\left.-\left(U\left(\vec{q}_{1}\right)-\left(\nabla U\left(\vec{q}_{1}\right), \vec{\xi}\right)\right) \delta t\right)\right\}\left(\psi\left(\vec{q}_{1}, \vec{q}_{0} ; t\right)-(\nabla \psi, \vec{\xi})+\nabla^{2} \psi \cdot \xi^{2} / 2\right) D^{3} \xi .
\end{aligned}
$$

First, we consider terms enveloped by braces (a) and (b): (a) here displacement $\left(\vec{\delta}_{1, k}-\vec{\delta}_{x, k}\right)$ divided by $\delta t$ is a velocity $\vec{v}_{k}$ of kth atom at its deviation from a steady position relative to the center of mass. Summation through all deviations of atoms divided by $N$ gives averaged velocity, $\vec{v}$, of all atoms with respect to the center of mass. This averaged velocity, as we mentioned above, vanishes. The velocity can be nonzero only in a case when external forces push coherently all atoms. This case we do not consider here. (b) this term is a thermal kinetic energy, $T_{N}$, of the atoms oscillating about the center of mass. Observe that energy of thermal fluctuations, $T_{N}$, is proportional to $k_{\mathrm{B}} T$. Because of its presence in the propagator intensity of an interference pattern diminishes in general. Further we shall add this term into the potential energy as some constant component.

In the light of the above observation we rewrite Eq. (12) as follows

$$
\begin{aligned}
\psi\left(\vec{q}_{1}, \vec{q}_{0} ; t\right)+\frac{\partial \psi}{\partial t} \delta t & =-\frac{1}{A} \int_{R^{3}} \exp \left\{-\frac{m_{N}}{2 \Gamma} \frac{\xi^{2}}{\delta t}\right\}(1+\underbrace{m_{N} \vec{v}}_{(a)} \frac{\vec{\xi}}{\Gamma}+\underbrace{T_{N}}_{(b)} \frac{\delta t}{\Gamma}+\left(U\left(\vec{q}_{1}\right)-\left(\nabla U\left(\vec{q}_{1}\right), \vec{\xi}\right)\right) \frac{\delta t}{\Gamma}) \\
& \times\left(\psi\left(\vec{q}_{1}, \vec{q}_{0} ; t\right)-(\nabla \psi, \vec{\xi})+\nabla^{2} \psi \cdot \xi^{2} / 2\right) D^{3} \xi .
\end{aligned}
$$

Here we have expanded preliminarily exponents to the Taylor series up to the first term of the expansion. Exception relates to the term $\exp \left\{-m_{N} \xi^{2} / 2 \Gamma \delta t\right\}$ which remains in its original form. This exponent integrated over all space $\mathrm{R}^{3}$ results in

$$
-\frac{1}{A} \int_{R^{3}} \exp \left\{-\frac{m_{N}}{2 \Gamma} \frac{\xi^{2}}{\delta t} D^{3} \xi=-\frac{1}{A}\left(\frac{2 \pi \Gamma \delta t}{m_{N}}\right)^{3 / 2}=1 \quad \Rightarrow \quad A=-\left(\frac{2 \pi \Gamma \delta t}{m_{N}}\right)^{3 / 2} .\right.
$$

To derive outcomes of integration of terms containing factors $(\nabla \psi, \vec{\xi})$ and $\nabla^{2} \psi \cdot \xi^{2} / 2$ we mention the following integrals [8]

$$
\frac{1}{A} \int_{R^{3}} \exp \left\{-\frac{m_{N}}{2 \Gamma} \frac{\xi^{2}}{\delta t}\right\} \xi D^{3} \xi=0 \quad \text { and } \quad \frac{1}{A} \int_{R^{3}} \exp \left\{-\frac{m_{N}}{2 \Gamma} \frac{\xi^{2}}{\delta t}\right\} \xi^{2} D^{3} \xi=\frac{\Gamma}{m_{N}} \delta t
$$


In the light of this observation let us now solve integral (13) accurate to terms containing $\delta t$ not higher the first order:

$$
\psi\left(\vec{q}_{1}, \vec{q}_{0} ; t\right)+\frac{\partial \psi}{\partial t} \delta t=\psi\left(\vec{q}_{1}, \vec{q}_{0} ; t\right)+\frac{\Gamma}{2 m_{N}} \nabla^{2} \psi \delta t+\frac{1}{\Gamma}\left(U\left(\vec{q}_{1}\right)-T_{N}\right) \psi \delta t .
$$

We note that the term $(\vec{v}, \nabla \psi)$ here is absent since we consider $\vec{v}=0$, as was mentioned above. By reducing from the both sides the function $\psi\left(\vec{q}_{1}, \vec{q}_{0} ; t\right)$ we come to the following differential equation

$$
\frac{\partial \psi\left(\vec{q}_{1}, \vec{q}_{0} ; t\right)}{\partial t}=\frac{\Gamma}{2 m_{N}} \nabla^{2} \psi\left(\vec{q}_{1}, \vec{q}_{0} ; t\right)+\frac{1}{\Gamma}\left(U\left(\vec{q}_{1}\right)-T_{N}\right) \psi\left(\vec{q}_{1}, \vec{q}_{0} ; t\right) .
$$

The parameter

$$
\frac{\Gamma}{2 m_{N}}=\frac{2 k_{\mathrm{B}} T \delta t}{2 m_{N}}+\mathbf{i} \frac{\hbar}{2 m_{N}}
$$

is seen to be as a complex-valued diffusion coefficient consisting of real and imaginary parts.

\subsubsection{Temperature is zero}

Let $k_{\mathrm{B}} T \delta t=0$. It means that $\Gamma=\mathrm{i} \hbar$. Also $T_{N}=0$. One can see that Eq. (17) is reduced to

$$
\mathbf{i} \hbar \frac{\partial \psi\left(\vec{q}_{1}, \vec{q}_{0} ; t\right)}{\partial t}=-\frac{\hbar^{2}}{2 m_{N}} \Delta \psi\left(\vec{q}_{1}, \vec{q}_{0} ; t\right)+U\left(\vec{q}_{1}\right) \psi\left(\vec{q}_{1}, \vec{q}_{0} ; t\right) .
$$

It is the Schrödinger equation.

\subsubsection{Temperature is not zero}

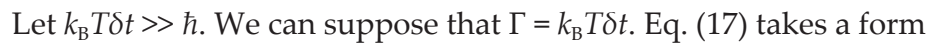

$$
\frac{\partial \psi\left(\vec{q}_{1}, \vec{q}_{0} ; t\right)}{\partial t}=D \Delta \psi\left(\vec{q}_{1}, \vec{q}_{0} ; t\right)+\frac{\left(U\left(\vec{q}_{1}\right)-T_{N}\right)}{2 m_{N} D} \psi\left(\vec{q}_{1}, \vec{q}_{0} ; t\right) .
$$

Here 


$$
D=\frac{k_{\mathrm{B}} T \delta t}{m_{N}}
$$

is the diffusion coefficient. The coefficient has dimensionality of [length ${ }^{2}$ time $\left.^{-1}\right]$. It is a factor of proportionality representing amount of substance diffusing across a unit area in a unit time - concentration gradient in unit time.

We can see that Eq. (20) deals with the amplitude function $\psi$, not a concentration. However, a measurable function is $\rho=|\psi|^{2}$ - concentration having dimensionality of [(amount of substance) length ${ }^{-3}$. Let us multiply Eq. (20) from the left by $2 \psi$. First we note that the combination $\quad 2 \psi \Delta \psi=2 \psi \nabla \psi^{-1} \psi \nabla \psi=2\left(\psi \nabla \psi^{-1}\right)(\psi \nabla \psi)+2 \nabla(\psi \nabla \psi) \quad$ results in $-(1 / 2)(\nabla \ln (\rho) \cdot \nabla \rho)+\Delta \rho$. As a result we come to a diffusion-drift equation describing diffusion in a space loaded by a potential field $\left(U\left(\vec{q}_{1}\right)-T_{N}\right)$ :

$$
\frac{\partial \rho}{\partial t}+\frac{D}{2}(\nabla \ln (\rho), \nabla \rho)=D \Delta \rho+\frac{\left(U\left(\vec{q}_{1}\right)-T_{N}\right)}{2 m_{N} D} \rho
$$

Extra term $(D / 2) \nabla \ln (\rho)$ in this diffusion-drift equation is a velocity of outflow of the particles from volume populated by much more number of particles than in adjacent volume. The term $-\ln (\rho)$ is entropy of a particle ensemble. From here it follows that $\nabla \ln (\rho)$ describes inflow of the particles to a region where the entropy is small. Observe that the velocity

$$
\vec{u}=D \frac{\nabla \rho}{\rho}=D \nabla \ln (\rho)
$$

is an osmotic velocity required of the particle to counteract osmotic effects [6]. Namely, imagine a suspension of many Brownian particles within a physical volume acted on by an external, virtual in general, force. This force is balanced by an osmotic pressure force of the suspension [6]:

$$
\vec{K}=k_{\mathrm{B}} T \frac{\nabla \rho}{\rho} .
$$

From here it is seen, that the osmotic pressure force arises always when density difference exists and especially when the density tends to zero. And vice-versa, the force disappears in extra-dense media with spatially homogeneous distribution of particles. As states the second law of thermodynamics spontaneous processes happen with increasing entropy. The osmosis evolves spontaneously because it leads to increase of disorder, i.e., with increase of entropy. When the entropy gradient becomes zero the system achieves equilibrium, osmotic forces vanish. 
Due to appearance of the term $(D / 2) \nabla \ln (\rho)$ in Eq. (22) the diffusion equation becomes nonlinear. It is interesting to note, running ahead, that this osmotic term reveals many common with the quantum potential, which is show further. Observe that the both expressions contain the term $\nabla \rho / \rho=\nabla \ln (\rho)$ relating to gradient of the quantum entropy $S_{Q}=-\ln (\rho) / 2$ [28].

Reduction to PDEs, Eq. (19) and Eq. (22), was done with aim to show that the both quantum and classical realms adjoin with each other much more closely, than it could seem with the first glance. Further we shall return to the integral path paradigm [8] and calculate patterns arising after passing particles through gratings. We shall combine quantum and classical realms by introducing the complex-valued parameter $\Gamma=2 k_{\mathrm{B}} T \delta t+\mathrm{i} \hbar$.

\subsection{Paths through $N$-slit grating}

Computation of a passing particle through a grating is based on the path integral technique [8]. We begin with writing the path integral that describes passing the particle through a slit made in an opaque screen that is situated perpendicularly to axis $z$, Fig. 3. For this reason we need to describe a movement of the particle from a source to the screen and its possible deflection at passing through the slit, see Fig. 4. At that we need to evaluate all possible deflections.

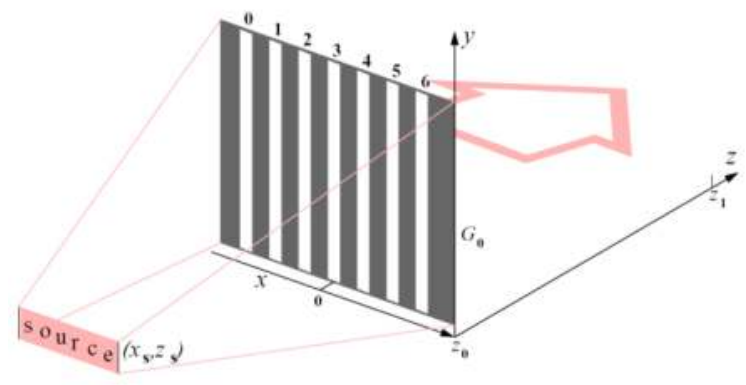

Figure 3. Interferometry from one grating $G_{0}$ situated transversely to a particle beam emitted from a distributed source.

We believe, that before the screen and after it, the particle (fullerene molecule, for example) moves as a free particle. Its Lagrangian, rewritten from Eq. (17), describes its deflection from a straight path in the following form

$$
L=m_{N} \frac{\dot{x}^{2}}{2}-m_{N}(\dot{x}, \vec{v})+T_{N}
$$

The first term relates to movement of the center of mass of the molecule. So that $m_{N}$ is mass of the molecule and $\dot{x}$ is its transversal velocity, i.e., the velocity lies in transversal direction to the axis $z$. The second term is conditioned by collective fluctuations of atoms constituent 
this molecule. This term is nonzero when atoms have predominant fluctuations along axis $x$. For the sake of simplicity we admit that $\vec{v}$ is constant. The third term is a constant and comes from Eq. (17). It can be introduced into the normalization factor. For that reason we shall ignore this term in the following computations. A longitudinal momentum, $p_{z}$ is much greater than its transverse component $[16,17,29]$ and we believe it is constant also. By translating a particle's position on a small distance $\delta x=\left(x_{\mathrm{b}}-x_{\mathrm{a}}\right)<<1$ for a small time $\delta t=\left(t_{\mathrm{b}}-t_{\mathrm{a}}\right)<<1$ we find that a weight factor of such a translation has the following form

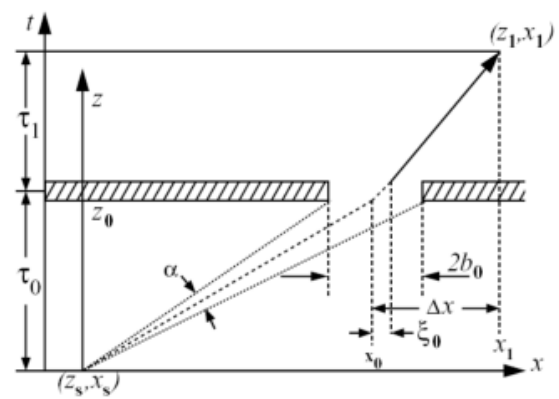

Figure 4. Passage of a particle along path $\left(z_{s}, x_{s}\right) \rightarrow\left(z_{0}, x_{0}\right) \rightarrow\left(z_{1}, x_{1}\right)$ through a screen containing one slit with a width equal to $z_{b 0}$. Divergence angle of particles incident on the slit, $a$, tends to zero as the source is removed to infinity.

$$
\exp \left\{-\frac{L \delta t}{\Gamma}\right\}=\exp \left\{-\frac{m_{N}}{2 \Gamma} \frac{\left(x_{b}-x_{a}\right)^{2}}{\left(t_{b}-t_{a}\right)}+\frac{m_{N} v}{\Gamma}\left(x_{b}-x_{a}\right)\right\}
$$

The particles flying to the grating slit along a ray $\alpha$, Fig. 4 , pass through the slit within a range from $x_{0}-b_{0}$ to $x_{0}+b_{0}$ with high probability. The path integral in that case reads

$$
\psi\left(x_{1}, x_{0}, x_{s}\right)=\int_{-b_{0}}^{b_{0}} K\left(x_{1}, \tau_{0}+\tau_{1} ; x_{0}+\xi_{0}, \tau_{0}\right) K\left(x_{0}+\xi_{0}, \tau_{0} ; x_{s}, 0\right) d \xi_{0} .
$$

Integral kernel (propagator) for the particle freely flying is as follows [8, 26]

$$
K\left(x_{b}, t_{b} ; x_{a}, t_{a}\right)=\left[\frac{m_{N}\left(1-2 v / v_{(a, b)}\right)}{2 \pi \Gamma\left(t_{b}-t_{a}\right)}\right]^{1 / 2} \exp \left\{-\frac{m_{N}\left(x_{b}-x_{a}\right)^{2}}{2 \Gamma\left(t_{b}-t_{a}\right)}\left(1-2 \frac{v}{v_{(a, b)}}\right)\right\}
$$

Here $v_{(a, b)}=\left(x_{b}-x_{a}\right) /\left(t_{b}-t_{b}\right)$ is a velocity of the molecule on a segment from $x_{a}$ to $x_{b}$. And $v$ is an average velocity of collective deflection of atoms constituent the molecule. It was defined 
in Eq. (12). We shall believe that the ratio $v / v_{(a, b)}$ is small enough. We can define a new renormalized mass $\{m\}_{N}=m_{N}\left(1-v / v_{(a, b)}\right)$ and further we shall deal with this mass.

\subsubsection{Passing of a particle through slit}

By substituting the kernel (28) into the integral (27) we obtain the following detailed form

$$
\psi\left(x_{1}, x_{0}, x_{s}\right)=\int_{-b_{0}}^{b_{0}}\left(\frac{\{m\}_{N}}{2 \pi \Gamma \tau_{1}}\right)^{1 / 2} \exp \left\{-\frac{\{m\}_{N}\left(x_{1}-\left(x_{0}+\xi_{0}\right)\right)^{2}}{2 \Gamma \tau_{1}}\right\}\left(\frac{\{m\}_{N}}{2 \pi \Gamma \tau_{0}}\right)^{1 / 2} \exp \left\{-\frac{\{m\}_{N}\left(\left(x_{0}+\xi_{0}\right)-x_{s}\right)^{2}}{2 \Gamma \tau_{0}}\right\} d \xi_{0} .
$$

The integral is computed within a finite interval $\left[-b_{0},+b_{0}\right]$. Observe, that the integrating can be broadened from $-\infty$ to $+\infty$. But in this case we need to load the integral by the step function equal to unit within the finite interval $\left[-b_{0},+b_{0}\right]$ and it vanishes outside of the interval. The step function, that simulate a single slit, can be approximated by the following a set of the Gaussian functions [30]

$$
G(\xi, b, \eta, K)=\frac{1}{\eta} \sqrt{\frac{2}{\pi}} \sum_{k=1}^{K} \exp \left\{-\frac{(K \xi-b(K-(2 k-1)))^{2}}{2(b \eta)^{2}}\right\}
$$

Here parameter $b$ is a half-width of the slit, real $\eta>0$ is a tuning parameter, and $K$ takes integer values. At $K=1$ this form-factor degenerates to a single Gaussian function. And at $K \rightarrow \infty$ this function tends to an infinite collection of the Kronecker deltas which fill everywhere densely the step function. We rewrite Eq. (29) with inserting this form-factor

$$
\psi\left(x_{1}, x_{0}, x_{s}\right)=\int_{-\infty}^{\infty} G\left(\xi_{0}, b_{0}\right) \frac{\{m\}_{N}}{2 \pi \Gamma \sqrt{\tau_{1} \tau_{0}}} \exp \left\{-\frac{\{m\}_{N}}{2 \Gamma}\left(\frac{\left(x_{1}-\left(x_{0}+\xi_{0}\right)\right)^{2}}{\tau_{1}}+\frac{\left(\left(x_{0}+\xi_{0}\right)-x_{s}\right)^{2}}{\tau_{0}}\right)\right\} d \xi_{0}
$$

We do not write parameters $\eta$ and $K$ in the Gaussian form-factor and for the sake of simplicity further we shall consider they equal to 1 . That is, for simulating the slit we select a single Gaussian function.

\subsubsection{Definition of new working parameters}

First we replace the flight times $\tau_{0}$ and $\tau_{1}$ by flight distances $\left(z_{0}-z_{\mathrm{s}}\right)$ and $\left(z_{1}-z_{0}\right)$, see Fig. 4 . This replacement reads

$$
\left\{\begin{array}{l}
\tau_{0}=\left(z_{0}-z_{s}\right) / v_{z} \\
\tau_{1}=\left(z_{1}-z_{0}\right) / v_{z}
\end{array}\right.
$$


where $v_{z}$ is a particle velocity along the axis $z$.

There is, however, one more parameter of time that is represented in definition of the coefficient $\Gamma=2 k_{\mathrm{B}} T \delta t+\mathrm{i} \hbar$. It is a small time increment $\delta t$. The parameter $\delta t$ first appears in the path integral (3) as the time increment along a path. In accordance with the uncertainty principle $\delta$ should be more or equal to the ratio of $\hbar$, Planck constant, to energy of occurring events. In a case of a flying particle through vacuum it can be minimal energy of vacuum fluctuations (it is about energy of the first Bohr orbit of electron-positron pair that is about $14 \mathrm{eV}$ ). Evaluation gives $\delta t \sim 2.810^{-16} \mathrm{~s}$. From here it follows, that $2 k_{\mathrm{B}} T \delta t$ is less than $\hbar$ on about one order at $T=1000 \mathrm{~K}$ (almost temperature of fullerene evaporation from the Knudsen cell [27]).

Emergence of the term $2 k_{\mathrm{B}} T \delta t$ can be induced by existence of quantum drag [31] owing to different conditions for quantum fluctuations both inside of the fullerene molecule and outside what can induce weak Casimir forces. Because of the weak Casimir force the quantum drag does not lead to decoherence at least in the near zone. However further we shall see that a weak washing out of the Talbot interference pattern is due to existence of this term.

Let us divide the parameter $\Gamma$ by $\{m\}_{N} v_{z}$

$$
\frac{\Gamma}{\{m\}_{N} v_{z}}=\frac{2 k_{\mathrm{B}} T}{\{m\}_{N} v_{z}} \delta t+\mathbf{i} \frac{\hbar}{\{m\}_{N} v_{z}} .
$$

Here $p_{z}=\{m\}_{N} v_{z}$ is a particle momentum along axis $z$. We can define the de Broglie wavelength $\lambda_{\mathrm{dB}}=h / p_{z}$ where $h=2 \pi h$ is the Planck constant. Let us also define a length $\delta_{T}=$ $4 \pi k_{\mathrm{B}} T \delta t /\left(\{m\}_{N} v_{z}\right)$. In this view we can rewrite Eq. (33) as follows

$$
\frac{\Gamma}{\{m\}_{N} v_{z}}=\frac{\delta_{T}}{2 \pi}+\mathbf{i} \frac{\lambda_{\mathrm{dB}}}{2 \pi}=\frac{1}{2 \pi} \Lambda .
$$

The length $\delta_{T}$ tends to zero as $T \rightarrow 0$. At $T=1000 \mathrm{~K}$ and at adopted $\delta t=2.810^{-16} \mathrm{~s}$ we have $\delta_{T}$ $\approx 0.4 \mathrm{pm}$. On the other hand, the de Broglie wavelength, $\lambda_{\mathrm{dB}}$, evaluated for the fullerene molecule moving with the velocity $v_{z}=100 \mathrm{~m} / \mathrm{s}$ is about $5 \mathrm{pm}$ [18]. So, we can see that the length $\delta_{T}$ is less of the de Broglie wavelength on about one order and smaller. A signification of the length $\delta_{T}$ is that it determines decoherence of a particle beam. Decoherence of flying particles occurs the quickly, the larger $\delta_{T}$. Observe that the length $\delta_{T}$ has a close relation with the coherence width - a main parameter in the generalized Gaussian Schell-model [32, 33].

\section{Wave function behind the grating}

Wave function from one slit after integration over $\xi_{0}$ from $-\infty$ to $+\infty$ has the following view [28] 


$$
\psi\left(x, z, x_{0}, x_{s}\right)=\sqrt{\frac{2}{\pi \Lambda \Sigma_{0}\left(z_{0}-z_{s}\right)}} \exp \left\{-\frac{\pi}{\Lambda}\left[\frac{\left(x-x_{0}\right)^{2}}{\left(z-z_{0}\right)}\left(1-\frac{\Xi_{0}^{2}}{\Sigma_{0}}\right)+\frac{\left(x_{0}-x_{s}\right)^{2}}{\left(z_{0}-z_{s}\right)}\right]\right\} .
$$

Here argument of $\psi$-function contains apart $x$ also $z$ in order to emphasize that we carry out observation in the point $(z, x)$, see Fig. 4 . The factor $(2 / \pi)^{1 / 2}$ comes from (30). Parameters $\Xi_{0}$ and $\Sigma_{0}$ read

$$
\Xi_{0}=1-\frac{\left(x_{0}-x_{s}\right)\left(z-z_{0}\right)}{\left(z_{0}-z_{s}\right)\left(x-x_{0}\right)} \quad \text { and } \quad \Sigma_{0}=\frac{\left(z-z_{s}\right)}{\left(z_{0}-z_{s}\right)}+\frac{\Lambda\left(z-z_{0}\right)}{2 \pi b_{0}^{2}}
$$

In order to simplify records here we omit subscript 1 at $x$ and $z-$ an observation point that is situated after the slit.

Let us consider that an opaque screen contains $N_{0}$ slits spaced through equal distance, $d$, from each other. Numeration of the slits is given as it is shown in Fig. 3, $n_{0}=0,1,2, \ldots, N_{0}-1$. Sum of all wave functions (35), each of which calculates outcome from an individual slit, gives a total effect in the point $(z, x)$ where a detector is placed:

$$
\left|\Psi_{0}\left(x, z, d, x_{s}, \Lambda\right)\right\rangle=\sum_{n_{0}=0}^{N_{0}-1} \psi\left(x, z,\left(n_{0}-\frac{N_{0}-1}{2}\right) d, x_{x}\right)
$$

Probability density in the vicinity of the observation point $(x, z)$ reads

$$
p(x, z)=\left\langle\Psi_{0}\left(x, z, d, x_{s}, \Lambda\right) \mid \Psi_{0}\left(x, z, d, x_{s}, \Lambda\right)\right\rangle .
$$

Calculation of the wave function (37) is fulfilled for the grating containing $N_{0}=32$ slits. Distance between slits is $d=10^{5} \lambda_{\mathrm{dB}}$. So at $\lambda_{\mathrm{dB}}=5 \mathrm{pm}$ the distance is equal to $500 \mathrm{~nm}$. Requirement $\lambda_{\mathrm{dB}}<<d$ and $N_{0}$ tending to infinity together with a condition that the particle beam is paraxial, that is, $x_{\mathrm{s}}=0$ and $z_{\mathrm{s}} \rightarrow-\infty$, provides emergence in the near-field of an interference pattern, named Talbot carpet $[19,20]$. Here a spacing along interference patterns is measured in the Talbot length

$$
z_{\mathrm{T}}=2 \frac{d^{2}}{\lambda_{\mathrm{dB}}}
$$

which is a convenient natural length at representation of interference patterns. Since we restrict themselves by finite $N_{0}$ we have a defective carpet, which progressively collapses as a 
spacing from the slit increases. Fig. 5 shows the Talbot carpet, being perfect in the vicinity of the grating slit; it is destroyed progressively with increasing $z_{\mathrm{T}}$. As for the Talbot carpet we have the following observation. We see that in a cross-section $z=z_{\mathrm{T}} / 2$ image reproduces radiation of the slits but phase-shifted by half period between them. At $z=z_{\mathrm{T}}$ radiation of the slits is reproduced again on the same positions where the slits are placed. And so forth.

Evaluation of sizes of the interference pattern is given by ratio of the Talbot length to a length of the slit grating. In our case the Talbot length is $z_{\mathrm{T}}=0.1 \mathrm{~m}$. And length of the slit grating is about $N_{0} d=1.610^{-5} \mathrm{~m}$. From here we find that the ratio is 6250 . It means that the interference pattern shown in Fig. 5 represents itself a very narrow strip.

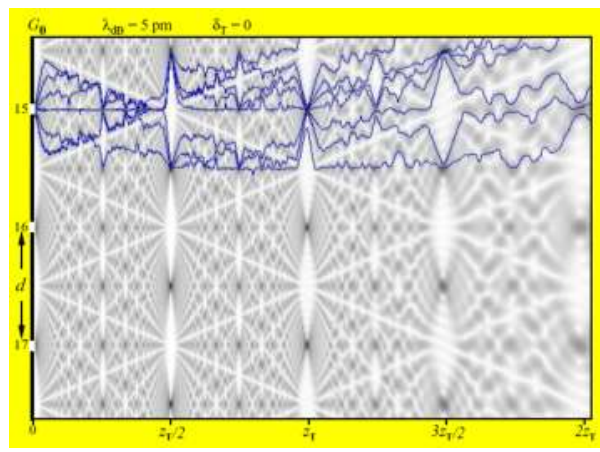

Figure 5. Interference pattern in the near field. It is shown only the central part of the grating containing $N_{0}=32$ slits, $\lambda_{\mathrm{dB}}=5 \mathrm{pm}, d=500 \mathrm{~nm}$, and $\delta_{\mathrm{T}}=0$. In the upper part of the figure a set of the Bohmian trajectories, looking like on zigzag curves, drawn in dark blue color is shown.

Zigzag curves, drawn in the upper part of Fig. 5 by dark blue color, show Bohmian trajectories that start from the slit No. 15. One can see that particles prefer to move between nodes having positive interference effect and avoid empty lacunas. However the above we noted, that the ratio of the Talbot length to the length of the grating is about $6250>>1$. It means that really the Bohmian trajectories look almost as straight lines slightly divergent apart. Zigzaglike behavior of the trajectories is almost invisible. Such an almost feebly marked zigzag-like behavior may be induced by fluctuations of virtual particles escorting the real particle.

As soon as we add the term $k_{\mathrm{B}} T \delta t$ different from zero $(T>0 \mathrm{~K})$ we observe blurring the interference pattern. The blurring is the stronger, the larger $k_{\mathrm{B}} T \delta t$. For comparison see Figs. 6 and 7. Here instead of $k_{\mathrm{B}} T \delta t$ we write a more convenient parameter, the coherence length $\delta_{\mathrm{T}}$. This length characterizes a dispersed divergence from initially tuned the de Broglie wavelength. Such a disperse medium can be due to quantum drag on the vacuum fluctuations. Here the Bohmian trajectories are not shown, since because of the thermal term $k_{\mathrm{B}} T \delta t>0$ a Brownian like scattering of the trajectories arises. This scattering we shall discuss later on. 


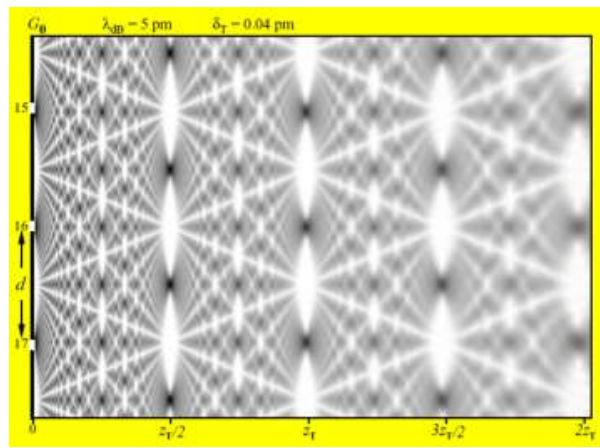

Figure 6. Blurred interference pattern in the near field. It is shown only the central part of the grating containing $N_{0}=$ 32 slits; $d=500 \mathrm{~nm}, \delta_{\mathrm{T}}=0.04 \mathrm{pm} \ll<\lambda_{\mathrm{dB}}=5 \mathrm{pm}$.

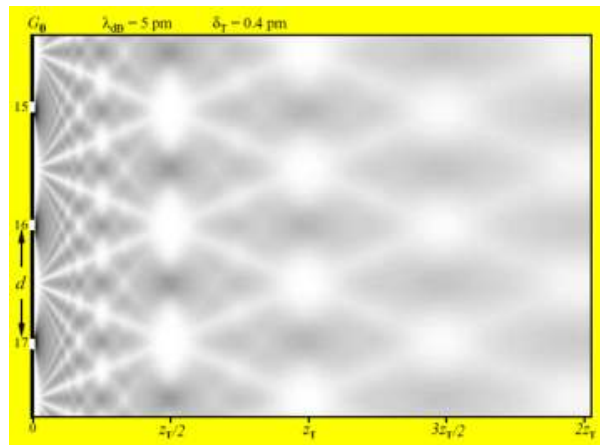

Figure 7. Blurred interference pattern in the near field. It is shown only the central part of the grating containing $N_{0}=$ 32 slits; $d=500 \mathrm{~nm}, \delta_{\mathrm{T}}=0.4 \mathrm{pm}<\lambda_{\mathrm{dB}}=5 \mathrm{pm}$.

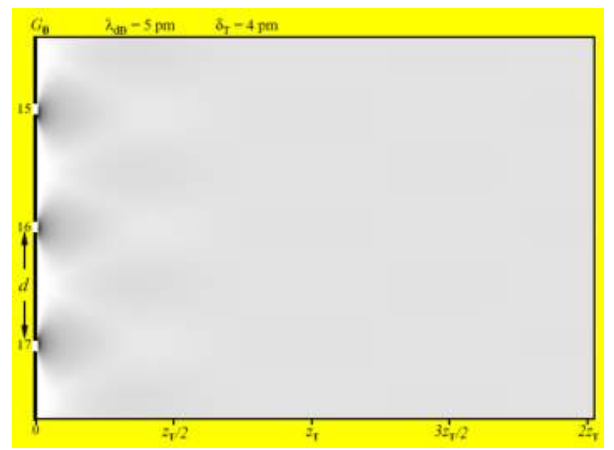

Figure 8. Destroyed interference pattern because of large $\delta_{\mathrm{T}}=4 \mathrm{pm} \sim \lambda_{\mathrm{dB}}=5 \mathrm{pm} . N_{0}=32$ slits, $d=500 \mathrm{~nm}$. 
We may think that the technical vacuum can be not perfect. It causes additional scattering of particles on residual gases. Because of this additional scattering the interference pattern can be destroyed entirely, as shown in Fig. 8.

Now let us draw dependence of the probability density $p(x, z)$ as a function of $x$ at fixed $z$. In other words, we calculate interference fringes in a cross-section of the interference patterns at $z=z_{\mathrm{T}} / 2$ for different values of the length $\delta_{\mathrm{T}}$. Such a cross-section is chosen because a self image of the slit grating appears phase-shifted by half period of the grating. For that reason we should see the interference fringes spaced between the slit sources of radiation.

Fig. 9 shows three characteristic patterns of the interference fringes. In Fig. 9(a) almost ideal interference fringes are shown obtained at $\delta_{\mathrm{T}}<\lambda_{\mathrm{dB}}$. Fig. 9(b) shows interference fringes obtained at $\delta_{\mathrm{T}} \sim \lambda_{\mathrm{dB}}$. It is instructive to compare these interference fringes with those that have been measured in experiments [14,34]. And Fig. 9(c) shows disappearance of interference fringes because of strong scattering of the particles on residual gases in vacuum, $\delta_{\mathrm{T}}>\lambda_{\mathrm{dB}}$.
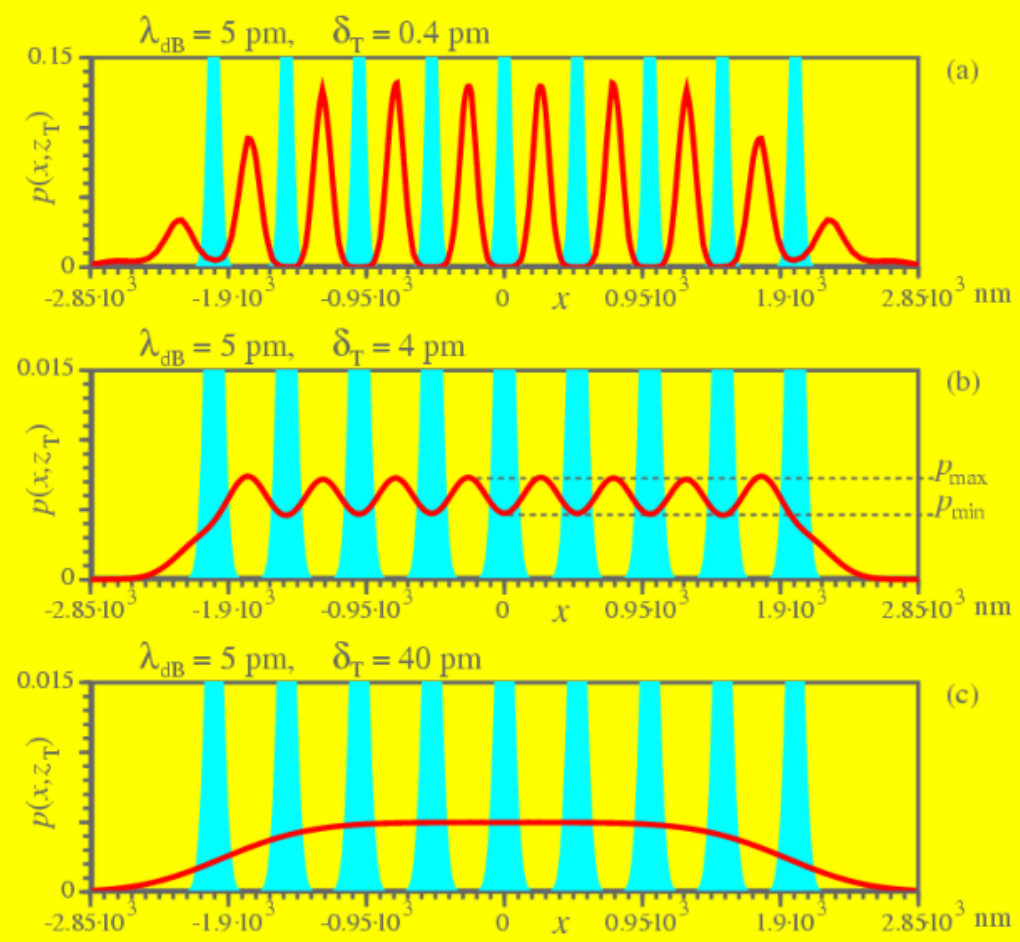

Figure 9. Interference fringes in cross-section of the density distribution pattern by the Talbot half-length, $z=z_{\mathrm{T}} / 2$, (the fringes are drawn in red): (a) $\delta_{T}=0.4 \mathrm{pm}$, almost coherent beam; (b) $\delta_{T}=4 \mathrm{pm}$, weak coherence; (c) $\delta_{\mathrm{T}}=40 \mathrm{pm}$, entirely noncoherent beam. Cyan strips show luminosity of slits. The grating consists of $N_{0}=9$ slits. Collapse of the interference pattern on edges of the grating is due to its finite length. Therefore visibility of the interference fringes is evaluated only for 5 central slits. 
Disappearance of interference fringes is numerically evaluated by calculating a characteristic called visibility [14, 34]. The fringe visibility [27] is represented as a ratio of difference between maximal and minimal intensities of the fringes to their sum:

$$
V=\frac{P_{\max }-P_{\min }}{P_{\max }+P_{\min }}
$$

Evaluation of $P_{\max }$ and $P_{\min }$ is shown in Fig. 9(b). As follows from the figure, the evaluations are fulfilled in a central region of the grating. That is, edges of the grating have to be left far off from the measured zone. The visibility $V$ as a function of the parameter $\delta_{\mathrm{T}}$ is shown in Fig. 10. One can see that crossover from almost perfect interference fringes, $V=1$, up to their absence, $V=0$, begins near $\delta_{\mathrm{T}} \sim \lambda_{\mathrm{dB}}$. Transition from almost coherent particle beam to incoherent is a cause of such a crossover [30].

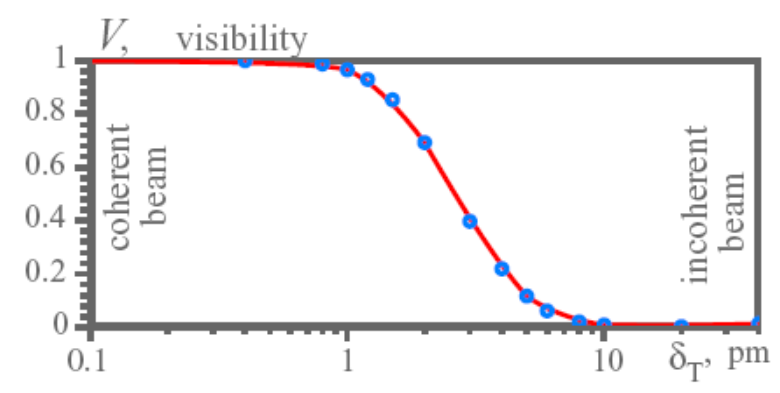

Figure 10. Visibility of interference fringes as a function of the parameter $\delta_{\top}$ ranging from 0.1 to 40 pm. Wavelength of a matter wave is $\lambda_{\mathrm{dB}}=5 \mathrm{pm}$.

\section{Bohmian trajectories and variance of momenta and positions along paths}

Here we repeat computations of David Bohm [35] which lead to the Hamiltoton-Jacobi equation loaded by the quantum potential and, as consequence, to finding Bohmian trajectories. But instead of the Schrödinger equation we choose Eq. (17) that contains complex-valued parameter $\Gamma=\beta+\mathrm{i} \hbar$ :

$$
(\beta-\mathbf{i} \hbar) \frac{\partial \psi}{\partial t}=\frac{\left(\beta^{2}+\hbar^{2}\right)}{2 m_{N}} \nabla^{2} \psi+\frac{(\beta-\mathbf{i} \hbar)}{(\beta+\mathbf{i} \hbar)} V \psi .
$$

Here $\beta=2 k_{\mathrm{B}} T \delta t$ (in particular, the diffusion coefficient reads $\left.D=\beta / 2 m_{N}\right)$ and $V=U\left(\vec{q}_{1}\right)-T_{N}$. 
Further we apply polar representation of the wave function, $\psi=R \exp \{\mathrm{iS} / \hbar\}$. It leads to obtaining two equations for real and imaginary parts that deal with real-valued functions $R$ and $S$. The function $R$ is the amplitude of the wave function and $S / \hbar$ is its phase. After series of computations, aim of which is to put together real and imaginary terms, we obtain the following equations

$$
\begin{gathered}
\frac{\partial S}{\partial t}+\beta \frac{1}{R} \frac{\partial R}{\partial t}=-\underbrace{\frac{\left(\beta^{2}+\hbar^{2}\right)}{2 m_{N} \hbar^{2}}(\nabla S)^{2}}_{(\mathrm{a})}+\underbrace{\frac{\left(\beta^{2}+\hbar^{2}\right)}{2 m_{N}} \frac{\nabla^{2} R}{R}}_{(\mathrm{b})}+\frac{\left(\beta^{2}-\hbar^{2}\right)}{\left(\beta^{2}+\hbar^{2}\right)} V, \\
\frac{\beta}{\hbar^{2}} \frac{\partial S}{\partial t}-\frac{1}{R} \frac{\partial R}{\partial t}=\frac{\left(\beta^{2}+\hbar^{2}\right)}{2 m_{N} \hbar^{2}} \underbrace{\left(\nabla^{2} S+2 \frac{1}{R} \nabla R \nabla S\right)}_{\text {(c) }}-\frac{2 \beta}{\left(\beta^{2}+\hbar^{2}\right)} V .
\end{gathered}
$$

Firstly, we can see that at $\beta=0$ Eq. (42) reduces to the modified Hamilton-Jacobi equation due to loaded the quantum potential that is enveloped here by brace (b). And Eq. (43) reduces to the continuity equation. These equations read

$$
\begin{gathered}
\frac{\partial S}{\partial t}=-\underbrace{\frac{1}{2 m_{N}}(\nabla S)^{2}}_{(\mathrm{a})}+\underbrace{\frac{\hbar^{2}}{2 m_{N}} \frac{\nabla^{2} R}{R}}_{(\mathrm{b})}+V, \\
-\frac{1}{R} \frac{\partial R}{\partial t}=\frac{1}{2 m_{N}} \underbrace{\left(\nabla^{2} S+2 \frac{1}{R} \nabla R \nabla S\right)}_{(\mathrm{c})} .
\end{gathered}
$$

Terms enveloped by braces (a), (b), and (c) are the kinetic energy of the particle, the quantum potential, and the right part is a kernel of the continuity equation (45), respectively. In particular, the term $2 \nabla R / R=\nabla \ln \left(R^{2}\right)$ relates to the osmotic velocity, see Eq. (23). Eqs. (44) and (45) are the same equations obtained by Bohm [35]. From historical viewpoint it should be noted that the same equations were published by Madelung ${ }^{1}$ in 1926 [36].

Momentum of the particle reads

$$
\vec{p}=m_{N} \vec{v}=\nabla S,
$$

where $\vec{v}$ is its current velocity. The de Broglie equation relates the momentum $p$ to the wavelength $\lambda_{\mathrm{dB}}=h / p$, where $h=2 \pi h$ is the Planck constant. Now, as soon as we found the current velocity 


$$
\vec{v}=\frac{1}{m_{N}} \nabla S=\frac{\hbar}{m_{N}} \operatorname{Im}\left(|\psi\rangle^{-1} \nabla|\psi\rangle\right)
$$

positions of the particle in each current time beginning from the grating' slits up to a detector is calculated by the following formula

$$
\vec{r}(t+\delta t)=\vec{r}(t)+\vec{v}(t) \delta t
$$

Here $t$ is a current time that starts from $t=0$ on a slit source and $\delta t$ is an arbitrarily small increment of time. Some calculated trajectories of particles, the Bohmian trajectories, are shown in upper part of Fig. 5. It should be noted that the Bohmian trajectories follow from exact solutions of Eqs. (44)-(45). These equations give a rule for finding geodesic trajectories and secants of equal phases, $S / \hbar$, at given boundary conditions. The geodesic trajectories point to tendency of the particle migration along paths. And the secant surfaces describe a coherence of all the passing particles created on a single source.

In case of $\beta \gg$ $\hbar$ we have the following two equations

$$
\begin{gathered}
\frac{\partial R}{\partial t}=-\underbrace{\frac{\beta}{2 m_{N} \hbar^{2}} R(\nabla S)^{2}}_{(\mathrm{a})}+\underbrace{\frac{\beta}{2 m_{N}} \nabla^{2} R}_{(\mathrm{b})}, \\
\frac{\partial S}{\partial t}=\frac{\beta}{2 m_{N}} \underbrace{\left.\nabla^{2} S+2 \frac{1}{R} \nabla R \nabla S\right)}_{(\mathrm{c})},
\end{gathered}
$$

Here we take into consideration that in the first equation we may replace $(\nabla S / \hbar)^{2}=4 \pi^{2} / \lambda_{\mathrm{dB}}^{2}$. In the second equation we may replace the term $\nabla S$ by $m_{N} \vec{v}$ as follows from Eq. (46). We notice also, that $2 R^{-1} \nabla R=2 \nabla \ln (R)=\nabla \ln (\rho)$. And $\beta / 2 m_{N}=D$ is the diffusion coefficient. Now we may rewrite Eqs. (49)-(50) as follows

$$
\begin{gathered}
\frac{\partial R}{\partial t}=\underbrace{D \nabla^{2} R}_{(\mathrm{b})}-\underbrace{\frac{4 \pi^{2}}{\lambda_{\mathrm{dB}}^{2}} D R}_{\text {(a) }}, \\
\frac{\partial S}{\partial t}=D \underbrace{\left(\nabla^{2} S+m_{N}(\nabla \ln (\rho), \vec{v})\right)}_{\text {(c) }}=D \nabla^{2} S+m_{N}(\vec{u}, \vec{v}) .
\end{gathered}
$$


Here $\vec{u}=D \nabla \ln (\rho)$ is the osmotic velocity defined in Eq. (23). We got the two diffusion equations coupled with each other through sources. Namely, this coupling is provided due to the de Broglie wavelength and the osmotic velocity which can change with time. These diffusion equations cardinally differ from Eqs. (44)-(45). Because of diffusive nature of these supplementary parts blurring of interference patterns occurs. It leads to degeneration of the Bohmian trajectories to Brownian ones.

\subsection{Dispersion of trajectories and the uncertainty principle}

As for the Bohmian trajectories there is a problem concerning their possible existence. As follows from Eqs. (46) and (48) in each moment of time there are definite values of the momentum and the coordinate of a particle moving along the Bohmian trajectory. This statement enters in conflict with the uncertainty principle.

Here we try to retrace emergence of the uncertainty principle stemming from standard probability-theoretical computations of expectation value and variance of a particle momentum. We adopt a wave function in the polar representation

$$
|\Psi\rangle=\operatorname{Rexp}\{\mathbf{i} S / \hbar\}
$$

where $R=(\rho)^{1 / 2}$ is the amplitude of the wave function $\left(\rho=R^{2}=\langle\Psi \mid \Psi\rangle\right.$ is the probability density) and $S / \hbar$ is its phase. Momentum operator $\hat{p}=-i \hbar \nabla$ and corresponding velocity operator $\hat{v}=-\boldsymbol{i}(\hbar / m) \nabla$ are kinetic operators in quantum mechanics. Here $m$ is mass of the particle. Expectation value of the velocity operator reads

$$
\vec{V}_{g}=\frac{1}{\langle\Psi \mid \Psi\rangle}\left\langle\Psi\left|-\mathbf{i} \frac{\hbar}{m} \nabla\right| \Psi\right\rangle=\frac{1}{m}\left(\nabla S+\mathbf{i} \hbar \nabla S_{Q}\right) .
$$

The velocity $\mathrm{V}_{\mathrm{g}}$ is seen to be complex-valued. Here $S_{Q}=-\ln (R)=-\ln (\rho) / 2$ is the quantum entropy [28] and $(\hbar / 2 m)$ is the quantum diffusion coefficient [6, 7]. Therefore its imaginary part is a quantum osmotic velocity

$$
\vec{u}_{Q}=-(\hbar / m) \nabla S_{Q}=(\hbar / 2 m) \nabla \ln (\rho) .
$$

It is instructive to compare this velocity with the classical osmotic velocity given in Eq. (23). As can see the osmotic velocity stems from gradient of entropy that evaluates degree of order and disorder on a quantum level, likely of vacuum fluctuations.

Real part of Eq. (54) gives the current velocity $\vec{v}$ defined by Eq. (47). It should be noted that because of existence of imaginary unit in definition of the momentum operator, real part of Eq. (54) is taken as the current velocity. Whereas imaginary unit is absent in Eq. (47). Therefore at computing the current velocity by Eq. (47) we take imaginary part. 
Let us now calculate variance of the velocity $\mathrm{V}_{\mathrm{g}}$. This computation reads

$$
\operatorname{Var}\left(V_{g}\right)=\frac{1}{\langle\Psi \mid \Psi\rangle}\left\langle\Psi\left|\left(-\mathbf{i} \frac{\hbar}{m} \nabla-\vec{V}_{g}\right)^{2}\right| \Psi\right\rangle=\frac{1}{\langle\Psi \mid \Psi\rangle}\left\langle\Psi|\mathbf{i} \frac{\hbar}{m} \nabla \vec{V}_{g}+\underbrace{\mathbf{i} \frac{\hbar}{m} \vec{V}_{g} \nabla+V_{g}^{2}}_{(\mathrm{d})}-\frac{\hbar^{2}}{m^{2}} \Delta| \Psi\right\rangle
$$

Terms over bracket (d) kill each other as follows from Eq. (54). It is reasonable in the perspective to multiply $\operatorname{Var}\left(\mathrm{V}_{\mathrm{g}}\right)$ by $m / 2$

$$
\frac{m}{2} \operatorname{Var}\left(V_{g}\right)=-\frac{1}{\langle\Psi \mid \Psi\rangle}\left\langle\Psi\left|\frac{\hbar^{2}}{2 m} \Delta\right| \Psi\right\rangle+\mathbf{i} \frac{\hbar}{2} \nabla \vec{V}_{g}
$$

So, this expression has a dimensionality of energy. The first term to be computed represents the following result

$$
-\frac{1}{\langle\Psi \mid \Psi\rangle}\left\langle\Psi\left|\frac{\hbar^{2}}{2 m} \Delta\right| \Psi\right\rangle=\underbrace{\frac{1}{2 m}(\nabla S)^{2}}_{\text {(a) }}-\underbrace{\frac{\hbar^{2}}{2 m} \frac{\nabla^{2} R}{R}}_{\text {(b) }}-\mathbf{i} \frac{\hbar}{2 m} \underbrace{\left(\Delta S+2 \frac{(\nabla S, \nabla R)}{R}\right)}_{\text {(c) }} .
$$

Here the term enveloped by bracket (a) is a kinetic energy of the particle, the term enveloped by bracket (b) with negative sign added is the quantum potential $Q$, and the term enveloped by bracket (c) comes from the continuity equation. See for comparison Eqs. (44) and (45). We rewrite the quantum potential as follows

$$
Q=-\frac{\hbar^{2}}{2 m} \frac{\nabla^{2} R}{R}=-\frac{\hbar^{2}}{2 m}\left(\nabla S_{Q}\right)^{2}+\frac{\hbar^{2}}{2 m} \nabla^{2} S_{Q}
$$

As for the second term in Eq. (57) we have $i(\hbar / 2) \nabla \vec{V}_{g}=i(\hbar / 2 m) \Delta S-\left(\hbar^{2} / 2 m\right) \Delta S_{Q}$. It is follows from computation by Eq. (54). As a result, the expression (57) takes the following view

$$
\frac{m}{2} \operatorname{Var}\left(V_{g}\right)=\frac{1}{2 m}(\nabla S)^{2}-\frac{\hbar^{2}}{2 m}\left(\nabla S_{Q}\right)^{2}-\mathbf{i} \frac{\hbar}{m} \frac{(\nabla S, \nabla R)}{R}
$$

One can see that the variance consists of real and imaginary parts. Observe that the right side is represented through square of gradient of the complexified action [28], namely $\left(\nabla\left(S+\mathrm{i} \hbar S_{Q}\right)\right)^{2} / 2 m$. We shall not consider here the imaginary part. Instead we shall consider the real part of this expression. It reads 


$$
\frac{m}{2} \operatorname{Re}\left(\operatorname{Var}\left(V_{g}\right)\right)=\frac{1}{2 m}(\nabla S)^{2}-\hbar\left(\frac{\hbar}{2 m}\left(\nabla S_{Q}\right)^{2}\right)
$$

The first term in this expression represents kinetic energy, $E$, of the particle. The second term, stemming from the quantum potential, contains under braces a term having dimensionality of inverse time, that is, of frequency

$$
\omega_{Q}=\frac{\hbar}{2 m}\left(\nabla S_{Q}\right)^{2}
$$

This frequency multiplied by $\hbar$ represents an energy binding a particle with vacuum fluctuations. This energy, as follows from Eq. (61), is equal to the particle mass multiplied by squared the osmotic velocity (55) and divided by 2 . It is an osmotic kinetic energy. In the light of the above said we rewrite Eq. (61) in the following way

$$
\frac{m}{2} \operatorname{Re}\left(\operatorname{Var}\left(V_{g}\right)\right)=E-\hbar \omega_{Q} \geq 0
$$

Let we have two Bohmian trajectories. Along one trajectory we have $E_{1}-\hbar \omega_{Q, 1}$, and along other trajectory we have a perturbed value $E_{2}-\hbar \omega_{Q, 2}$. Subtracting one from other we have

$$
\delta E-\hbar \delta \omega_{Q} \geq 0
$$

One can suppose that emergence of the second trajectory was conditioned by a perturbation of the particle moving along the first trajectory. If it is so, then emergence of the second trajectory stems from an operation of measurement of some parameters of the particle. One can think that duration of the measurement is about $\delta t=1 / \delta \omega_{Q}$. From here we find

$$
\delta E \delta t \geq \hbar
$$

Now let us return to Eq. (48) and rewrite it in the following view

$$
\delta \vec{r}(t)=\vec{v}_{1}(t) \delta t \geq \vec{v}_{1}(t) \hbar / \delta E .
$$

The initial Bohmian trajectory is marked here by subscript 1 . Observe that $\delta E=m\left(v_{2}^{2}-v_{1}^{2}\right) / 2 \approx m \vec{v}_{1} \delta \vec{v}$. Here we have calculated $v_{2}^{2}=\left(\vec{v}_{1}+\delta \vec{v}\right)^{2} \approx v_{1}^{2}+2 \vec{v}{ }_{1} \delta \vec{v}$. Substituting computations of $\delta E$ into Eq. (66) we obtain finally 


$$
\delta \vec{p} \delta \vec{r} \geq \hbar
$$

Here we take into account $\delta \vec{p}=m \delta \vec{v}$.

\section{Concluding remarks}

Each nanoparticle incident on a slit grating passes only through a single slit. Its path runs along a Bohmian trajectory which is represented as an optimal path for the nanoparticle migrating from a source to a detector. Unfortunately, the Bohmian trajectory can not be observable since a serious obstacle for the observation comes from the uncertainty principle. In other words, an attempt to measure any attribute of the nanoparticle, be it position or orientation, i.e., the particle momentum, leads to destroying information relating to future history of the nanoparticle. What is more, any collision of the nanoparticle with a foreign particle destroys the Bohmian trajectory which could give a real contribution to the interference pattern. It relates closely with quality of vacuum. In the case of a bad vacuum such collisions will occur frequently. They lead to destruction of the Bohmian trajectories. Actually, they degenerate to Brownian trajectories.

Excellent article [21] of Couder \& Fort with droplets gives, however, a clear hint of what happens when the nanoparticle passes through a single grating slit. In the light of this hint we may admit that the particle "bouncing at moving through vacuum" generates a wave at each bounce. So, a holistic quantum mechanical object is the particle + wave. Here the wave to be generated by the particle plays a role of the pilot-wave first formulated by Lui de Broglie and later developed by Bohm [37]. It is interesting to note in this context, that the pilotwaves have many common with Huygens waves [5].

A particle passing through vacuum generates waves with wavelength that is inversely proportional to its momentum (it follows from the de Broglie formula, $\lambda=h / p$, where $h$ is Planck's constant). One can guess that a role of the vacuum in the experiment of Couder \& Fort takes upon itself a silicon oil surface with subcritical Faraday ripples activated on it [21]. Observe that pattern of the ripples is changed in the vicinity of extraneous bodies immersed in the oil which simulate grating slits. Interference of the ripples with waves generated by the bouncing droplets provides optimal paths for the droplets traveling through the slits and further. As a result we may observe an interference pattern emergent depending on amount of slits in the grating and distance between them.

Now we may suppose that the subcritical Faraday ripples on the silicon oil surface simulate vacuum fluctuations. Consequently, the vacuum fluctuations change their own pattern near the slit grating depending on amount of slits and distance between them. We may imagine that the particle passing through vacuum (bouncing through, Fig. 11) initiates waves which interfere with the vacuum fluctuations. As a result of such an interference the particle moves along an optimal path - along the Bohmian trajectory. Mathematically the bounce is imitated by an exponential term $\exp \{\mathrm{i} S / \hbar\}$, where the angle $S / \hbar$ parametrizes the group of rotation 
given on a circle of unit radius. So, the path along which the particle moves is scaled by this unitary group, $\mathrm{U}(1)$, due to the exponential mapping of the phase $S / \hbar$ on the circle.

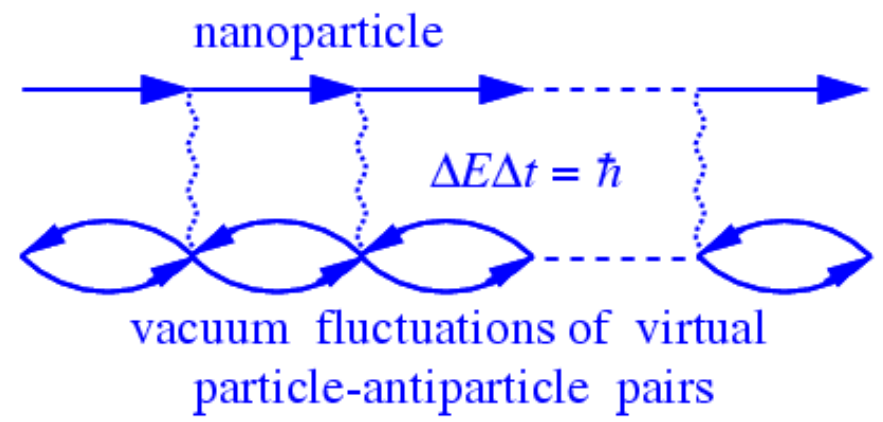

Figure 11. Bouncing a nanoparticle at moving through vacuum. Vertical dotted sinusoidal curves depict exchange by energy $\Delta E$ with vacuum virtual particle-antiparticle pairs over period of about $\Delta t=\hbar / \Delta E$.

In conclusion it would be like to remember remarkable reflection of Paul Dirac. In 1933 Paul Dirac drew attention to a special role of the action $S$ in quantum mechanics [38] - it can exhibit itself in expressions through $\exp \{\mathrm{iS} / \hbar\}$. In 1945 he emphasized once again, that the classical and quantum mechanics have many general points of crossing [39]. In particular, he had written in this article: "We can use the formal probability to set up a quantum picture rather close to the classical picture in which the coordinates $q$ of a dynamical system have definite values at any time. We take a number of times $t_{1}, t_{2}, t_{3}, \ldots$ following closely one after another and set up the formal probability for the q's at each of these times lying within specified small ranges, this being permissible since the q's at any time all commutate. We then get a formal probability for the trajectory of the system in quantum mechanics lying within certain limits. This enables us to speak of some trajectories being improbable and others being likely."

\section{Acknowledgement}

Author thanks Miss Pipa (administrator of Quantum Portal) for preparing programs that permitted to calculate and prepare Figures 5 to 8. Author thanks also O. A. Bykovsky for taking my attention to a single-particle interference observed for macroscopic objects by Couder and Fort and V. Lozovskiy for some remarks relating to the article.

\section{Author details}

Valeriy I. Sbitnev 
St.-Petersburg Nuclear Physics Institute, NRC Kurchatov Institute, Gatchina, Russia

\section{References}

[1] Dauben JW. Abraham Robinson: The creation of nonstandard analysis. A personal and mathematical odyssey. Princeton, NJ: Princeton University Press; 1995.

[2] Stanford Encyclopeia of Philosophy: Newton's Views on Space, Time, and Motion. http://plato.stanford.edu/entries/newton-stm/ (accessed 12 August 2004).

[3] Henson CW. Foundations of nonstandard analysis. In: Arkeryd L.O., Cutland N. J., \& Henson C. W., (eds.) Nonstandard Analysis, Theory and application. The Netherlands: Kluwer Acad. Publ.; 1997,p. 1-51.

[4] Nelson E. Radically Elementary Probability Theory. Princeton, New Jersey: Princeton Univ. Press; 1987.

[5] Huygens C. Treatise on Light. Gutenberg eBook, No. 14725. http://www.gutenberg.org/files/14725/14725-h/14725-h.h (accessed 18 January 2005)

[6] Nelson E. Dynamical theories of Brownian motion. Princeton, New Jersey: Princeton Univ. Press; 2001.

[7] Nelson E. Quantum fluctuations. Princeton Series in Physics. Princeon, New Jersey: Princeton Univ. Press; 2001.

[8] Feynman RP., Hibbs A. Quantum mechanics and path integrals. N. Y.: McGraw Hill; 1965.

[9] Einstein A., von Smoluchowski M. Brownian motion. Moscow-Leningrad: ONTI (in Russian); 1936.

[10] Sidorov LN., Troyanov SI. At the dawn of a new chemistry of fullerenes. PRIRODA 2011; 1153(9) 22-30.

[11] Arndt M., Hackermüller L., Reiger E. Interferometry with large molecules: Exploration of coherence, decoherence and novel beam methods. Brazilian Journal of Physics 2005; 35(2A) 216-223.

[12] Brezger B., Arndt M., Zeilinger A. Concepts for near-field interferometers with large molecules. J. Opt. B: Quantum Semiclass. Opt. 2003; 5(2) S82-S89.

[13] Brezger B., Hackermüller L., Uttenthaler S., Petschinka J., Arndt M., Zeilinger A. Matter-Wave Interferometer for Large Molecules. Phys. Rev. Lett. 2002; 88100404.

[14] Gerlich S., Eibenberger S., Tomandl M., Nimmrichter S., Hornberger K., Fagan P. J., Tuxen J., Mayor M., Arndt, M. Quantum interference of large organic molecules. Nature Communications 2011; (2) 263. http://www.nature.com/ncomms/journal/v2/n4/ full/ncomms1263.html (accessed 5 April 2011) 
[15] Hornberger K., Hackermüller L., Arndt M. Inuence of molecular temperature on the coherence of fullerenes in a near-field interferometer. Phys. Rev. A. 2005; 71, 023601.

[16] Hornberger K., Sipe JP., Arndt M. Theory of decoherence in a matter wave TalbotLau interferometer. Phys. Rev. A. 2004; 70, 053608.

[17] Nimmrichter S., Hornberger K. Theory of near-fieldmatter wave interference beyond the eikonal approximation. Phys. Rev. A. 2008; 78, 023612.

[18] Juffmann,T., Truppe S., Geyer P., Major AG., Deachapunya S., Ulbricht H., Arndt, M. Wave and particle in molecular interference lithography. Phys. Rev. Lett. 2009; 103, 263601.

[19] Berry M., Klein S. Integer, fractional and fractal Talbot effects, Journal of Modern Optics, 1996; 43(10) 2139-2164.

[20] Berry M., Marzoli L., Schleich W. Quantum carpets, carpets of light. Physics World, $2001 ;(6), 39-44$.

[21] Couder Y., Fort E. Single-Particle Diffraction and Interference at a Macroscopic Scale. Phys. Rev. Lett. 2006; 97 (6), 154101(4).

[22] Stratonovich RL. Topics in the theory of random noise. N.Y.: Gordon and Breach; 1963.

[23] Gnedenko BV. Theory of Probability. N.Y.: Gordon \& Breach; 1997.

[24] Ventzel AD. The course of stochastic processes theory. Moskow: Nauka (in Russian); 1975.

[25] Grosche C. An introduction into the Feynman path integral. http://arXiv.org/abs/hepth/9302097 (accessed 20 February 1993).

[26] MacKenzie R. Path integral methods and applications. http://arXiv.org/abs/quant-ph/ 0004090 (accessed 20 April 2000).

[27] Juffmann T., Milic A., Müllneritsch M., Asenbaum P., Tsukernik A., Tüxen J., Mayor M., Cheshnovsky O., Arndt, M. Real-time single-molecule imaging of quantum interference. Nature Nanotechnology - Letter. 2012; 7, 297-300. http://www.nature.com/ nnano/journal/v7/n5/full/nnano.2012.34.html?WT.ec_id=NNANO-201205 (accessed 25 March 2012)

[28] Sbitnev VI. Bohmian trajectories and the path integral paradigm - complexified Lagrangian mechanics. In: Pahlavani MR. (ed.) Theoretical Concepts of Quantum Mechanics. Rijeka: InTech; 2011 p313-340. http://www.intechopen.com/books/ theoretical-concepts-of-quantum-mechanics/the-path-integral-paradigm-and-bohmian-trajectories-from-the-lagrangian-mechanics-to-complexified-on (accessed 2004-2012)

[29] Nairz O., Arndt M., Zeilinger A. Quantum interference experiments with large molecules. Am. J. Phys. 2003; 71 (4), 319-325. 
[30] Sbitnev VI. Matter waves in the Talbot-Lau interferometry, http://arxiv.org/abs/ 1005.0890 (accessed 17 September 2010).

[31] Volokitin AI. Quantum drag and graphene. PRIRODA, 2011; 1153(9), 13-21.

[32] Mandel L., Wolf E. Optical coherence and quantum optics. Cambridge: Cambridge University Press; 1995.

[33] McMorran B.,Cronin AD. Model for partial coherence and wavefront curvature in grating interferometers. Phys. Rev. A. 2008; 78 (1), 013601(10).

[34] Gerlich S., Hackermüller L., Hornberger K., Stibor A., Ulbricht H., Gring M., Goldfarb F., Savas T., Müri M., Mayor M., Arndt, M. A Kapitza-Dirac-Talbot-Lau interferometer for highly polarizable molecules. Nature Physics. 2007; 3, 711-715.

[35] Bohm D. A suggested interpretation of the quantum theory in terms of "hidden variables", I \& II. Physical Review, 1952; 85, 166-179 \& 180-193.

[36] Madelung E. Quantentheorie in Hydrodynamischer form. Zts. f. Phys. 1926; 40, 322-326.

[37] Stanford Encyclopeia of Philosophy: Bohmian Mechanics. http://plato.stanford.edu/ entries/qm-bohm/ (accessed 26 October 2001).

[38] Dirac PAM. The Lagrangian in Quantum Mechanics. Physikalische Zeitschrift der Sowjetunion. 1933; 3, 64-72.

[39] Dirac PAM. On the analogy between classical and quantum mechanics. Rev. Mod. Phys. 1945; 17 (2 \& 3), 195-199. 
